\title{
Observations of Small Solar System Bodies with GAIA
}

\author{
Francois Mignard \\ OCA/CERGA, France
}

The ESA space astrometry mission has a great potential of observation and discovery on the solar system objects. GAIA is due for launch in 2010 and the survey mission will last five years. In addition to accurate astrometry of about 1 billion stars, the mission will survey all solar system objects brighter than 20th magnitude, in astrometry and with multicolor photometry. Each object will be observed about a hundred times during the five year mission. It is expected that more than 500000 asteroids will be repeatedly measured by the mission.

The main scientific output for the solar system physics will be a systematic search of NEOs, in particular inside the Earth orbit, a tremendous improvement in the orbit of the whole population of minor bodies, making all previous earthbased position almost obsolete in this respect, a full taxonomic classification made possible by the analysis of light over the 15 photometric bands, and the determination of $\sim 100$ masses of main belt objects thanks to the favorable close approaches during the period 2010-2015.

In this talk I will present the current status of the mission and the expected results for the solar system physics. 